\title{
ACOS - oder: ein beliebtes Streitthema
}

\author{
Das Asthma-COPD-Overlap-Syndrom ACOS stiftet unter Pneumologen weiter Unruhe. Viele möchten \\ den Begriff am liebsten streichen: Es sei kein Syndrom, sondern nur die Koexistenz zweier obstrukti- \\ ver Atemwegserkrankungen, wurde bei einem Symposium betont. Eigentlich eine eher akademische \\ Diskussion. Denn ob Syndrom oder Komorbidität: ACOS-Patienten haben ein deutlich höheres Exazer- \\ bationsrisiko als die nur mit Asthma und sollten früh erkannt und intensiv behandelt werden.
}

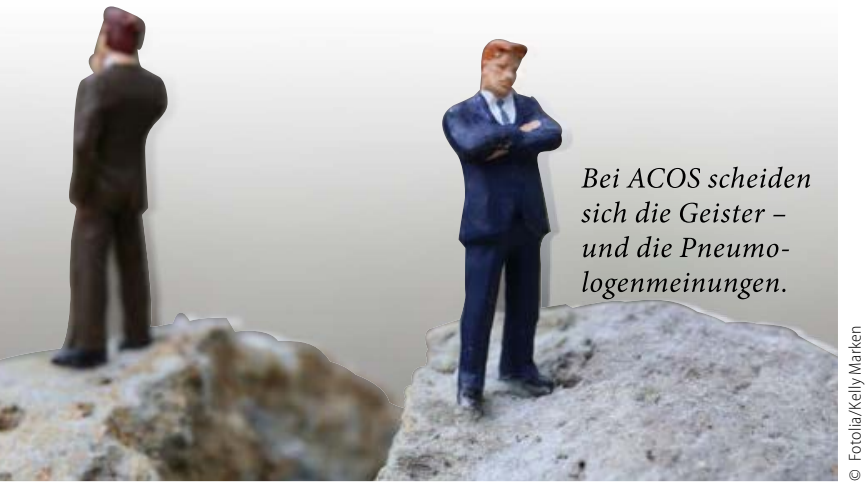

„ACOS sollte sterben!” ... „Dies ist kein Syndrom!”... „GOLD und GINA sollten ACOS aus den Leitlinien streichen!”... „Wir bewegen uns auf unbekanntem Terrain." Solche emotionalen Kommentare aus dem Auditorium bestimmten die Diskussion nach dem Vortrag von Dr. Tobias Bonten aus Leiden/Niederlande, in dem die Definition von ACOS bzw. besser der Versuch dessen im Fokus stand. Es gibt nämlich bis heute keine einheitliche Definition. Am meisten benutzt zur Charakterisierung der Patienten wird in der Literatur laut Bonten:

- Asthma und COPD, entweder nach eigenen Angaben der Patienten oder nach Diagnose beider Erkrankungen durch den Arzt.

- Manchmal wird zusätzlich der Nachweis einer irreversiblen Atemwegsobstruktion gefordert, gekennzeichnet durch den Parameter $\mathrm{FEV}_{1} / \mathrm{FVC}<0,7$, manchmal heißt es:

- $\mathrm{FEV}_{1} / \mathrm{FVC}<0,7$ und mehr als 10 Packungsjahre Raucheranamnese und Asthmadiagnose im Alter unter 40 Jahren,

- z.T. reicht statt der Asthmadiagnose ein FeNO-Wert > 45 ppb.

Je nach Definition variiert die Prävalenz von ACOS unter COPDbzw. Asthma-Patienten, betonte Bonten. In einer epidemiologischen Studie zu Übergewicht in den Niederlanden mit mehr als 5.600 Teilnehmern im Alter von 45-65 Jahren, darunter $850 \mathrm{~Pa}$ tienten mit Asthma/COPD, auf die die genannten Kriterien angewandt wurden, reichte die ACOS-Prävalenz von $10 \%$ bis zu $38 \%$. Gerade einmal ein Patient erfüllte alle ACOS-Definitionen, so Bonten. Für diese Erkenntnis gab es spontan Applaus aus dem Plenum, ein Signal für den Überdruss vieler Kollegen an ACOS. Ein weiterer Befund der Studie: Je länger ein Patient Asthma hatte, desto eher litt er nach eigenen Angaben auch unter ACOS. Bei der Bewertung der Klinik zeigte sich, dass das Vorliegen von ACOS, egal nach welchen Kriterien, mit der Prognose korrelierte: Die Exazerbationsrate war höher als bei Patienten, die nur Asthma hatten, während sich beim Vergleich mit COPD-Patienten allerdings nur geringe Unterschiede zeigten.

Die Häufigkeit von ACOS in einer Asthmakohorte und die Risikofaktoren wurden in einer schwedischen Studie mit insgesamt
2055 Asthma-Patienten und einem Beobachtungszeitraum von bis zu 30 Jahren untersucht. Die Teilnehmer hatten ärztlich diagnostiziertes Asthma oder erfüllten die typischen Asthma-Kriterien, so Dr. Helena Backman aus Umea. Lag zusätzlich postbronchodilatatorisch der $\mathrm{FEV}_{1} / \mathrm{FVC}$-Wert $<0$, 7, wurde ACOS diagnostiziert. 11\% der Teilnehmer erfüllten nach Angaben von Backman bei Studienbeginn die ACOS-Kriterien, 29\% im Verlauf der Studie. ACOS war häufiger bei Männern als bei Frauen (34\% vs. $25 \%)$ und wie zu erwarten auch häufiger bei Rauchern $(40 \%$ vs. 18\%) und bei älteren Studienteilnehmern. Übergewichtige hatten seltener ACOS. Klinische Unterschiede zwischen den Gruppen: ACOS-Patienten hatten gehäuft chronischen produktiven Husten ( $37 \%$ vs. $30 \%$ ) und persistierende pfeifende Atemgeräusche (35\% vs. $26 \%)$ und häufiger Schlafstörungen wegen wheezing oder Kurzatmigkeit als jene mit Asthma allein (50\% vs. $43 \%$ ). Auch bei den Komorbiditäten zeigten sich Auffälligkeiten: Asthma-Patienten hatten bei Studienbeginn öfter Atopien wie Heuschnupfen ( $42 \%$ vs. $31 \%$ ) und Ekzem (35\% vs. $28 \%$ ), während ACOS-Patienten etwas häufiger ischämische Herzerkrankungen hatten (13\% vs. $10 \%)$.

Roland Fath

Symposium „COPD and asthma COPDS overlap syndrome (ACOS): novelties in terms of prevalence, risk factors, and prognosis in COPD“, ERS-Kongress, London

\section{Häufiges Putzen kann die Lunge schädigen}

Wer beruflich in der Raumpflege arbeitet oder zu Hause regelmäßig für Sauberkeit sorgt, riskiert einen stärkeren Abfall der Lungenfunktion als der Altersdurchschnitt. Die beim Putzen eingeatmeten Dämpfe der Putzmittel haben offensichtlich respiratorische Relevanz. Bisher war bereits bekannt, dass im Beschäftigung im Putzdienst zu den Berufsgruppen mit dem höchsten COPD-Risiko gehört.

Nun berichten Autoren aus Norwegen über die Langzeitwirkungen der chronischen Putzmittelexposition. Sie hatten im Rahmen des European Community Respiratory Health Survey (ECRHS) über 20 Jahre die Lungenfunktion von knapp 3500 Frauen überwacht. Nach Adjustierung für Störvariable wiesen Frauen ohne Putzaktivitäten jährliche Abfällen der Lungenfunktionsparameter FVC und FEV , von 19,5 ml und 29,6 ml auf. Bei Frauen, die regelmäßig zuhause reinemachten, fielen diese Wert jährlich um 24,6 $\mathrm{ml}$ und $33,6 \mathrm{ml}$ ab. Hauptberufliche Putzfrauen wiesen eine durchschnittliche jährliche FVC- und FEV -Verluste von 27,6 ml und 34,6 ml auf. Die Unterschiede im Vergleich zu Frauen ohne Putzaktivitäten waren signifikant.

Fazit der Autoren: Wer regelmäßig putzt, weist ein erhöhtes Risiko für obstruktive bzw. restriktive Lungenerkrankungen auf. Betroffene sollten den Arzt aufsuchen, wenn sie Atembeschwerden verspüren. Putzsprays sollten mit Vorsicht eingesetzt werden.

ERS-Kongress 2016, 3.-7.9.2016 in London 